\title{
STRAW (STRings for Absorption length in Water): pathfinder for a neutrino telescope in the deep Pacific Ocean
}

\author{
Felix Henningsen ${ }^{1,2, *}$ for the STRAW Collaboration** \\ ${ }^{1}$ Physik-Department, Technische Universität München, D-85748 Garching, Germany \\ ${ }^{2}$ Max-Planck-Insitut für Physik, München, D-80805 München, Germany
}

\begin{abstract}
Recently, an opportunity for a pathfinder mission towards a new neutrino telescope appeared in the Pacific Ocean. An existing deep-sea electrooptical infrastructure maintained by Ocean Networks Canada could pose as the base towards a potential new large-volume neutrino telescope. Two groups from Technical University Munich and the University of Alberta pursued the optical characterization of the $2.6 \mathrm{~km}$ deep site called Cascada Basin within the scope of the "Strings for Absorption length in Water" (STRAW) project. We present a summary of the 1-year construction timeline, impressions of the detector performance and first results.
\end{abstract}

\section{Motivation}

The recent compelling evidence of a high-energy neutrino source [1][2][3] provides grounds for the further investigation of potential new neutrino telescopes on the planet. While the IceCube detector at the South Pole covers a relatively large fraction of the sky, a second telescope in the Northern Hemisphere is necessary to complement this coverage in neutrinos. While projects in the Mediterranean Sea as well in Lake Baikal in Siberia are being pursued, we investigate a potentially advantageous site in the Pacific Ocean.

The $2.6 \mathrm{~km}$ deep site called Cascadia Basin is located West off the coast of Vancouver Island and is part of the electro-optical research network called Ocean Networks Canada (ONC). This sub-facility of the University of Victoria provides a deep-sea network dedicated to experimental research. Its deep-sea and database maintenance is carried out by experts in the respective fields.

The first step towards a potential neutrino telescope in a new location is its characterization with respect to optical properties and possible backgrounds. Thus, as discussed in more detail in [4] and [5], the STRAW detector consists of a sensor-emitter array that will probe the absorption and scattering properties of the deep-sea waters. Furthermore, the sensor instruments will be able to assess potential backgrounds from bioluminescence and radioactivity. With a planned operation time of two years, the seasonal variations of these properties will be monitored.

\footnotetext{
*e-mail: felix.henningsen@tum.de

**e-mail: elisa.resconi@tum.de
} 


\section{Precision Optical Calibration Module (POCAM)}

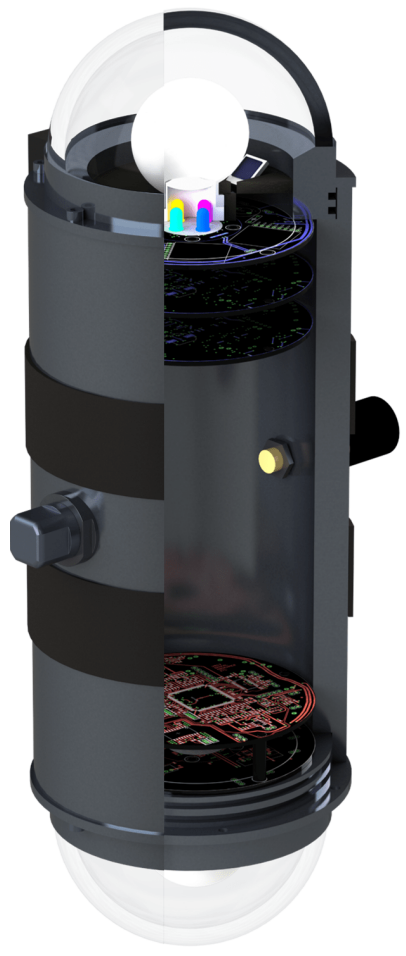

Figure 1. POCAM concept. [4]
The Precision Optical Calibration Module (POCAM) acts as an isotropic, in-situ calibrated light source instrument designed for large-volume neutrino telescope calibration [6]. It makes use of a semi-transparent teflon (PTFE) integrating sphere to isotropize light pulses from LEDs. The LED flashers are based on a modified version of the Kapustinsky LED pulsing circuit [7] which provides nanosecond light pulses.

The first POCAM protoype has been successfully installed and tested within the GVD neutrino telescope in spring of 2017. It was deployed within the detector $1000 \mathrm{~m}$ below the surface of Lake Baikal and several calibration runs were performed. Preliminary analyses confirm the expected attenuation length of the Baikal water which is also subject of ongoing research.

For the STRAW project, the POCAM was iterated and a second generation has been developed. This new version now hosts flashers of four different wavelengths covering the range of $365-600 \mathrm{~nm}$. Additionally, the light output characteristics of the flashers improved significantly both in intensity and timing as well as internal calibration precision. Furthermore, an automated setup has been built which allows for automatic calibration of the full dynamic range of a POCAM instrument. Further details on the characteristics of the second POCAM iteration can be found in [5].

\section{STRAW Detector}

From expectations [8] and previous measurements of deep waters [9][10], the absorption length in the deep-sea can reach maximum values of up to $60 \mathrm{~m}$ at $460 \mathrm{~nm}$ but reduces steeply towards smaller and larger wavelengths. To probe this primary quantity, the STRAW detector array hosts two $120 \mathrm{~m}$ long mooring lines, also called strings, with a total of three light emitters and five light sensors.

\subsection{Sensor-Emitter Array}

The geometry of the array puts instruments at 30,50,70 and $110 \mathrm{~m}$ height above the sea floor and the strings $40 \mathrm{~m}$ apart from each other. This not only provides light intensity baselines of $20-90 \mathrm{~m}$ and a fair amount of symmetry but is also the compromise of second-order technical limitations. While the former allows to probe the expected range of absorption lengths, the symmetry allows for more detailed studies of systematic effects at a later point.

The light emitter of the STRAW array is the isotropic POCAM, three of which are located on two strings. The light sensor for STRAW was a new development called STRAW Digital Optical Module (sDOM). It is based on the POCAM housing design but hosts two 3" photomultiplier tubes (PMTs) in each hemisphere. The PMTs are read-out using a Trigger Read-out Board (TRB3sc) and a PADIWA, both developed by GSI in Darmstadt which measures time-over-threshold (ToT) of the signal in multiple channels. The latter allows 
the reconstruction of charge and eventually the assessment of the absorption and scattering properties.

\subsection{Construction and Deployment}

The STRAW detector was designed, produced, calibrated and built on a timeline of eight months. In June 2018 the assembly was deployed at the Cascadia Basin site by ONC to its final depth of $2.6 \mathrm{~km}$ at which it is currently operating. After both strings were oriented towards each other by a remotely operated vehicle (ROV), they were connected to the ONC infrastructure and only minutes after, the detectors operational state was confirmed by ONC operators. However, we carried out first light measurements only several weeks after a complete system assessment which verified the system integrity.

\subsection{First Light Measurements}

For the first light measurements, we used the POCAM emission at different wavelengths and intensities to see how far the POCAM light would be seen. An example run is shown in Figure 2 using the time-difference between pulses that shows the detection of the POCAM $465 \mathrm{~nm}$-emission both at the minimum and maximum baseline of 20 and $90 \mathrm{~m}$, respectively. The detection over both baselines already provides promising grounds for the water transmission at Cascadia.

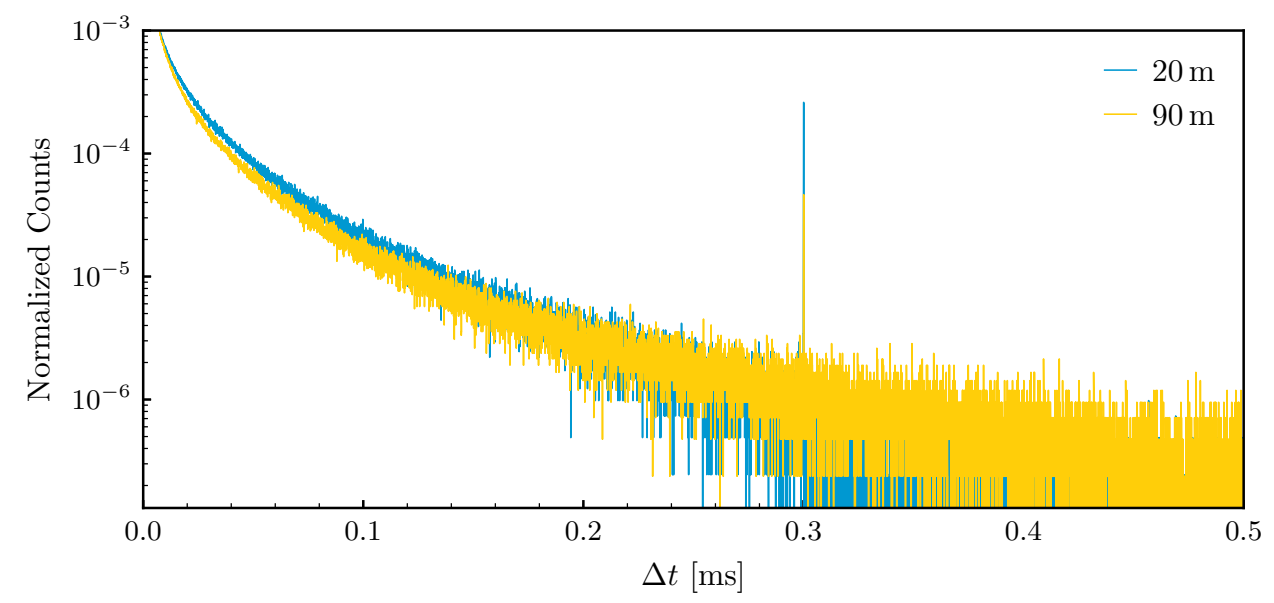

Figure 2. Time-difference histogram of subsequent pulses recorded by two sDOMs in STRAW with distances of 20 and $90 \mathrm{~m}$ to the flashing POCAM using $465 \mathrm{~nm}$ LED pulses. A clear signal at the flashing frequency of $3 \mathrm{kHz}$ confirms the detection over the full distance range of the STRAW detector. Adapted from [5].

Additionally, we used the sDOMs to record background from bioluminescence and radioactivity. An exemplary time profile of both short- and long-term monitoring is shown in Figure 3. The existence of biological and radioactive background is well consistent with other experiments in the deep sea [e.g. 11]. It is now a matter of further long-term monitoring and analysis to assess the feasibility of the Cascadia Basin site to host a neutrino telescope. Furthermore, we will invest more time into the investigation of the biological background 

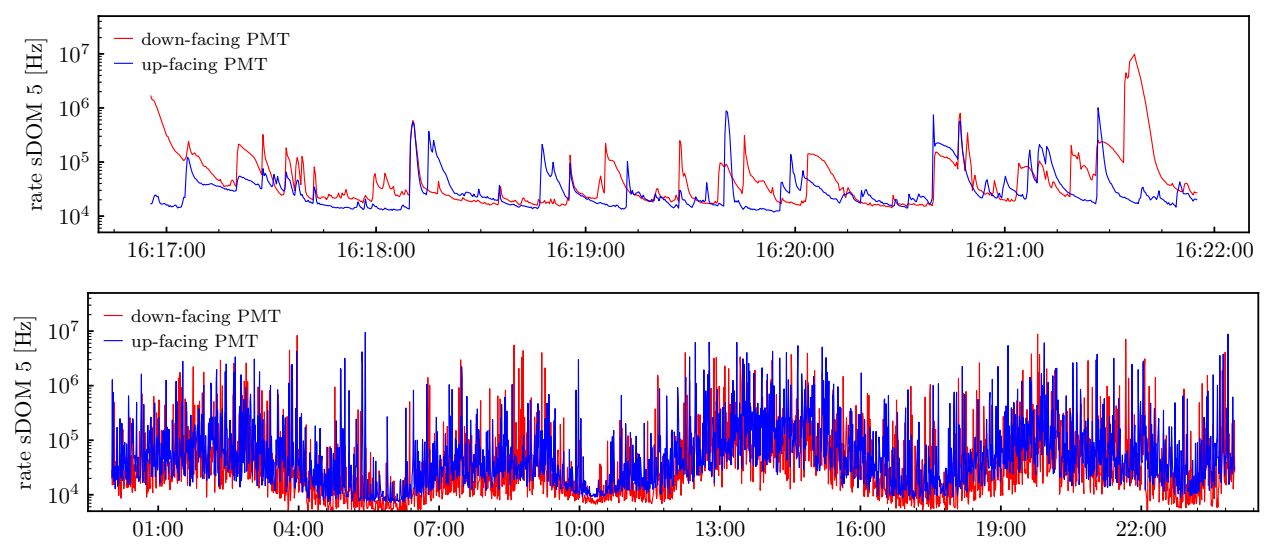

Figure 3. Dark rate of sDOM \#5 deployed at a depth of $2.6 \mathrm{~km}$ at the Cascadia Basin. The plots show a short term (top) and a long term (bottom) monitoring of the PMT dark rate in Hertz with respect to UTC time. Both time profiles show distinct structural features which are subject to further investigation. Adapted from [4].

\section{Summary and Outlook}

In collaboration with ONC, the STRAW project has successfully deployed two $120 \mathrm{~m}$ long mooring lines at $2.6 \mathrm{~km}$ depth in the Pacific Ocean. Furthermore, the ONC infrastructure and deep-sea procedures have verified the ability to handle, deploy and operate structures similar to neutrino telescope strings.

The future of STRAW is now two-fold. Firstly, further analyses and monitoring will assess the optical properties of the site in detail - in absorption, scattering and background radiation. Secondly, a potential upgrade of STRAW, called STRAW-b, will be realized in the season of 2019/2020 with the deployment of a $500 \mathrm{~m}$ long mooring line with further instrumentation to assess additional properties of the Cascadia Basin site.

\section{References}

[1] M.G. Aartsen et al. (Liverpool Telescope, MAGIC, H.E.S.S., AGILE, Kiso, VLA/17B403, INTEGRAL, Kapteyn, Subaru, HAWC, Fermi-LAT, ASAS-SN, VERITAS, Kanata, IceCube, Swift NuSTAR), Science 361, eaat1378 (2018), 1807.08816

[2] M.G. Aartsen et al. (IceCube), Science 361, 147 (2018), 1807.08794

[3] P. Padovani et al., Monthly Notices of the Royal Astronomical Society (2018)

[4] M. Boehmer et al. (STRAW) (2018), 1810. 13265

[5] F. Henningsen, Master's thesis, Munich, Tech. U. (2018-07-19)

[6] M. Jurkovič et al. (IceCube Gen2), EPJ Web Conf. 116, 06001 (2016)

[7] B.K. Lubsandorzhiev, Y.E. Vyatchin, JINST 1, T06001 (2006), physics/0410281

[8] C. Mobley, Light and Water: Radiative Transfer in Natural Waters (1994)

[9] R.C. Smith, K.S. Baker, Applied Optics 20, 177 (1981)

[10] A. Capone et al., Nucl. Instrum. Methods. Phys. Res. A 487, 423 (2002)

[11] P. Bagley et al. (KM3NeT), KM3NeT: Technical Design Report for a Deep-Sea Research Infrastructure in the Mediterranean Sea Incorporating a Very Large Volume Neutrino Telescope (2009) 